\title{
Zisheng Shenqi decoction ameliorates monosodium urate crystal-induced gouty arthritis in rats through anti-inflammatory and anti-oxidative effects
}

\author{
JIERU HAN ${ }^{1}$, YING XIE ${ }^{2}$, FANGYU SUI ${ }^{3}$, CHUNHONG LIU $^{1}$, XIAOWEI DU ${ }^{4}$, \\ CHENGGANG LIU ${ }^{5}$, XIAOLING FENG ${ }^{6}$ and DEYOU JIANG ${ }^{2}$ \\ Departments of ${ }^{1}$ Seasonal Febrile Diseases, ${ }^{2}$ Synopsis of The Golden Chamber and ${ }^{3}$ Chinese Materia Medica, \\ School of Basic Medical Sciences; ${ }^{4}$ Department of Pharmacognosy, School of Pharmacy; ${ }^{5}$ Department of Febrile Diseases, \\ School of Basic Medical Sciences; ${ }^{6}$ Department of Gynaecology, The First Affiliated Hospital, \\ Heilongjiang University of Chinese Medicine, Harbin, Heilongjiang 150040, P.R. China
}

Received July 29, 2015; Accepted June 27, 2016

DOI: $10.3892 / \mathrm{mmr} .2016 .5526$

\begin{abstract}
Based on traditional Chinese medicinal theories on gouty arthritis, Zisheng Shenqi decoction (ZSD), a novel Chinese medicinal formula, was developed due to its multiple functions, including reinforcing renal function, promoting blood circulation and relieving pain. In the present study, the effect of ZSD on monosodium urate (MSU) crystal-induced gouty arthritis in rats was investigated and the underlying mechanisms were examined. The data from these investigations showed that the injection of MSU crystals into the ankle joint cavity caused significant elevations in ankle swelling and inflammatory cell infiltration into the synovium, whereas these abnormal changes were markedly suppressed by oral administration of ZSD (40 mg/kg) for 7 days. Mechanically, ZSD treatment prevented MSU crystal-induced inflammatory responses, as evidenced by downregulation in the expression levels of NACHT domain, leucine-rich repeat and pyrin domain containing protein (NALP) 1 and NALP6 inflammasomes, decreased serum levels of tumor necrosis factor- $\alpha$ and interleukin-1 $\beta$, and inhibited activation of nuclear factor- $\kappa \mathrm{B}$. In addition, ZSD administration markedly enhanced the anti-oxidant status in MSU crystal-induced rats by the increase in the activities of superoxide dismutase and glutathione peroxidase, and the levels of reduced glutathione. These results indicated that ZSD effectively prevented MSU crystal-induced gouty arthritis via modulating multiple anti-oxidative and anti-inflammatory pathways, suggesting a
\end{abstract}

Correspondence to: Dr Deyou Jiang, Department of Synopsis of The Golden Chamber, School of Basic Medical Sciences, Heilongjiang University of Chinese Medicine, 24 Heping Road, Harbin, Heilongjiang 150040, P.R. China

E-mail: deyou_jiang@126.com

Key words: Zisheng Shenqi decoction, monosodium urate crystals, gouty arthritis, anti-inflammatory, anti-oxidative promising herbal formula for the prevention and treatment of gouty arthritis.

\section{Induction}

Gouty arthritis, a chronic inflammatory disease characterized by severe pain and swelling of one or more synovial joints, results from disordered nucleic acid metabolism and subsequent deposition of monosodium urate (MSU) crystals in the joints (1). During attacks of gouty arthritis, MSU crystals induce mass leukocyte infiltration into the joint cavity and are phagocytosed by monocytes/macrophages, resulting in membranolysis, the production of reactive oxygen species (ROS) and the release of lysosomal enzymes $(2,3)$. The NACHT domain, leucine-rich repeat and pyrin domain-containing protein (NALP) inflammasomes are important in the pathogenesis of gouty arthritis (4). Following activation by MSU crystals, NALP inflammasomes induce the conversion of pro-caspase-1 into active caspase-1, which further cleaves pro-interleukin (IL)-1 $\beta$ into the active form, IL-1 $\beta(5,6)$. Along with chemotactic factors, IL-1 $\beta$ can activate other pro-inflammatory cytokines, including tumor necrosis factor (TNF)- $\alpha$ and nuclear factor $(N F)-\kappa B$, which are critical for the initiation and propagation of the inflammatory response, and contribute to bone erosion and damage $(7,8)$. Accordingly, non-steroidal anti-inflammatory drugs, including naproxen and indomethacin, and corticosteroids, including adrenocorticotrophic hormone and colchicine, are currently used as first-line therapies for gouty arthritis (9). However, these drugs are associated with serious side effects, including renal toxicity, gastrointestinal toxicity, hepatic damage and myelosuppression (10). Therefore, identifying novel agents with improved efficacy and reduced toxicity for the treatment of gouty arthritis is warranted.

Traditional Chinese medicines (TCM), particularly traditional herbal formulas, have been widely accepted and applied to treat gouty arthritis in China in previous decades due to their multi-targeted and overall regulatory effects, and reduced adverse effects (11). According to the TCM theories, 
gouty arthritis belongs to 'Bi syndrome (bi zheng)', which is associated with phlegm, blood stasis, and toxic heat. Gouty arthritis is one of the most common rheumatic diseases, and is associated with redness, swelling, inflammation and pain (12). Additionally, based on modern Chinese medicine and clinical experience, renal insufficiency is considered to be the underlying cause of gouty arthritis (13-15). Therefore, treatment involving reinforcing renal function may be a rational and promising therapeutic method for the treatment of gouty arthritis.

Zisheng Shenqi decoction (ZSD), comprising eleven medicinal herbs (Table I), has been developed based on the above theories from a well-known Chinese herbal formula, Liuwei Dihuang (LWDH), which contains six commonly used herbs: Radix Rehmanniae Praeparata (Shu Di Huang), Cornus officinalis (Shan Zhu Yu), Poria cocos (Fu Ling), Cortex Moutan (Mu Dan Pi), Rhizoma Dioscoreae (Shao Yao) and Rhizoma Alismatis (Ze Xie) (16). LWDH dates back to the Qing Dynasty, and has traditionally been used for hepatic and renal insufficiency (17). Rhizoma Smilacis Glabrae (Tu Fu Ling) and Dioscorea septemloba (Bi Xie) have been reported to be effective in expelling wind and removing damp, increasing the excretion of uric acid and decreasing the content of uric acid in the blood $(18,19)$. In addition, Tu Fu Ling is also beneficial in easing joint movement (18). Achyranthes bidentatae (Niu Xi) is one of the most commonly used herbs in formulas prescribed for promoting blood circulation, removing blood stasis, and strengthening the muscles and bones (20). Semen Plantaginis (Che Qian $\mathrm{Zi}$ ) is a medicinal herb used extensively clinically with dieretic and antidiarrhoeal functions, and function in expelling phlegm (21). The ripe fruits of Amomum villosum Lour. (Sha Ren) are embodied in the Chinese pharmacopeia and recorded to effectively treat gouty arthritis, abdominal distension and fatigue associated with chronic gastritis, duodenitis or ulcerative colitis, and to promote appetite (22). ZSD is scientifically prepared using the classical formula of LWDH with the addition of Rhizoma Dmilacis Glabrae, Dioscorea septemloba, Achyranthes bidentatae, Semen Plantaginis and Amomum villosum. In the TCM theories, this combination is considered to be effective for the treatment of gouty arthritis due to its multiple functions, including reinforcing the renal function, promoting blood circulation and relieving pain. However, there is insufficient scientific evidence on the effects of ZSD against gouty arthritis. In the present study, an experimental MSU crystal-induced rat model was established to investigate the potential effects of ZSD on gouty arthritis. The possible underlying mechanisms associated with anti-inflammatory and anti-oxidative effects in ZSD-treated gouty arthritis rats were also examined.

\section{Materials and methods}

Animals. A total of 72 male Wistar rats $(200 \pm 20 \mathrm{~g})$ were purchased from Vital River Laboratory Animal Technology Co., Ltd. (Beijing, China). All animals were housed five per cage under controlled conditions (12 h light/dark cycle with a temperature of $22 \pm 1^{\circ} \mathrm{C}$ and a humidity of $40-60 \%$ ), fed with a standard laboratory chow and had free access to water for the duration of the experiment. All animal care and experimental procedures were approved by the Animal Care Ethics and Use Committee of Heilongjiang University of Chinese Medicine (Harbin, China) and performed in accordance with the guidelines of this Committee.

ZSD preparation. The 11 medicinal herbs of $\mathrm{ZSD}$, as shown in Table I, were obtained from the Chinese Pharmacy at the First Affiliated Hospital of Heilongjiang University of Chinese Medicine. The herbs were placed into a container for Chinese herb decoction and soaked in $4 \mathrm{v} / \mathrm{w}$ distilled water for $30 \mathrm{~min}$, boiled for $30 \mathrm{~min}$ and extracted twice. Following being combined and filtrated through a G4 filter, the extract solution was concentrated in a $60^{\circ} \mathrm{C}$ water bath to produce different concentrations of the crude drug (1,2 and $4 \mathrm{~g} / \mathrm{ml})$. The decoction was pasteurized, bottled and stored at $4^{\circ} \mathrm{C}$.

Synthesis of MSU crystals. Uric acid (0.8 g) was dissolved in $155 \mathrm{ml}$ double-distilled water containing $5 \mathrm{ml} \mathrm{NaOH}(1 \mathrm{M})$, and the $\mathrm{pH}$ was adjusted to 7.2 with HCL. The uric acid solution was cooled and stirred at room temperature, and then stored overnight at $4^{\circ} \mathrm{C}$ for crystal formation. Subsequently, the precipitate was filtered from the solution, dried at $70^{\circ} \mathrm{C}$ for $4 \mathrm{~h}$, ground into a fine powder, sifted with a 200 mesh metal screen, sterilized by heating at $180^{\circ} \mathrm{C}$ for $2 \mathrm{~h}$ and stored in sterile conditions. Prior to administration, the MSU crystals were suspended in sterile phosphate-buffered saline (PBS; $\mathrm{pH} 7.2)$ at $20 \mathrm{mg} / \mathrm{ml}(23)$.

Establishment of the MSU-induced gouty arthritis model and treatment schedule. Following 1 week of adaptation, the rats were randomly divided into the following six groups, each containing 12 animals: Normal control group, MSU (model) group, MSU + colchicine (Col; $0.28 \mathrm{mg} / \mathrm{kg}$ ) group, MSU + ZSD (10 mg/kg; L-ZSD) group, MSU + ZSD (20 mg/kg; M-ZSD) group and MSU + ZSD (40 mg/kg; $\mathrm{H}-\mathrm{ZSD})$ group. Prior to the administration of MSU, the rats in the Col group, as a positive control group, were orally administrated for 7 days with $0.28 \mathrm{mg} / \mathrm{kg} / \mathrm{d}$ Col (Xishuangbanna Pharmaceutical Co., Ltd., Yunnan, China). The animals in the ZSD groups were treated orally with ZSD once a day for 7 days, and the doses used were 10,20 and $40 \mathrm{mg} / \mathrm{kg}$, respectively. The control group and model group were administered with an equal volume of $0.9 \%$ saline $(10 \mathrm{ml} / \mathrm{kg})$ by gavage for 7 days. Subsequently, gouty arthritis was induced on day $7,1 \mathrm{~h}$ following treatment. The rats were anesthetized by intraperitoneally injecting $10 \%$ chloral hydrate $(3.5 \mathrm{ml} / \mathrm{kg})$, following which each of the rats in the MSU experimental groups were treated with $50 \mu \mathrm{l}$ MSU solution $(20 \mathrm{mg} / \mathrm{ml})$, which was injected into the left ankle joint cavity. Each animal in the control group received an injection of $50 \mu \mathrm{l}$ saline in the left ankle joint cavity (23). Following MSU injection for $24 \mathrm{~h}$, the circumference of the left ankle of each rat was measured at the same position using a tie-line method, and blood was collected for serum separation. The rats in all the groups were sacrificed by intraperitoneal injection with $10 \%$ chloral hydrate, and samples of synovial tissue were removed from the joints of each rat, 6 of which were flash-frozen in liquid nitrogen and stored at $-80^{\circ} \mathrm{C}$ for western blot analysis. The six remaining knee joint capsules were obtained and fixed in $4 \%$ paraformaldehyde buffer for histopathological examination of synovial tissues. 
Table I. Composition of Zisheng Shenqi decoction.

\begin{tabular}{|c|c|c|c|}
\hline Chinese name & Latin name & English name & Quantity (g) \\
\hline Shu Di Huang & Radix rehmanniae praeparata & Prepared rhizome of adhesive rehmannia & 20 \\
\hline Shan Yu Rou & Cornus officinalis & Common macrocarpium fruit & 20 \\
\hline $\mathrm{Fu}$ Ling & Poria cocos & Poria & 20 \\
\hline Mu Dan Pi & Cortex moutan & Tree peony bark & 15 \\
\hline Shan Yao & Rhizoma dioscoreae & Common yam rhizome & 20 \\
\hline Ze Xie & Rhizoma alismatis & Oriental waterplantain rhizome & 20 \\
\hline Sha Ren & Amoтum villosum & Fructus amomi & 10 \\
\hline Huai Niu Xi & Achyranthes bidentatae & Radix cyathulae & 15 \\
\hline Che Qian Zi & Semen plantaginis & Plantain seed & 30 \\
\hline Tu Fu Ling & Rhizoma smilacis glabrae & Glabrous greenbrier rhizome & 30 \\
\hline Bi Xie & Dioscorea septemloba & Sevenlobed yam rhizome & 20 \\
\hline
\end{tabular}

Histopathological examination. The paraformaldehyde-fixed rat knee joint capsules were dehydrated in an alcohol gradient, embedded in paraffin and cut in cross sections of $5 \mu \mathrm{m}$ using a rotary microtome (RM2235; Leica, Mannheim, Germany). The sections were then stained with hematoxylin and eosin (H\&E), and the histopathological changes of the synovial tissues were analyzed under an optical microscope (DP73; Olympus Corporation, Tokyo, Japan).

Enzyme-linked immunosorbent assays (ELISAs) for IL-1 $\beta$ and TNF- $\alpha$. The levels of IL- $1 \beta$ and TNF- $\alpha$ in the serum were determined using commercial Rat IL-1 $\beta$ and TNF- $\alpha$ ELISA kits (USCN Life Science, Inc., Wuhan, China), respectively, according to the manufacturer's protocols. Briefly, $100 \mu \mathrm{l}$ of the diluent standard or serum samples were added into the coated wells and incubated at $37^{\circ} \mathrm{C}$ for $2 \mathrm{~h}$. Following removal of the supernatant, $100 \mu \mathrm{l}$ biotinylated antibody solution was added and incubated for $1 \mathrm{~h}$ at $37^{\circ} \mathrm{C}$. Following washing three times, $100 \mu \mathrm{l}$ avidin-peroxidase complex solution was added and incubated for $1 \mathrm{~h}$ at $37^{\circ} \mathrm{C}$. Following washing, $90 \mu \mathrm{l}$ tetramethylbenzidine color solution was added and incubated in the dark for $30 \mathrm{~min}$ at $37^{\circ} \mathrm{C}$. Finally, $50 \mu \mathrm{l}$ stop solution was added to terminate the reaction, and the optical density at $450 \mathrm{~nm}$ was measured using a plate reader (ELX-800; Biotek, Winooski, VT, USA).

Measurement of serum levels of superoxide dismutase (SOD), glutathione peroxidase (GSH-Px) and reduced glutathione (GSH). The activities of SOD, GSH-Px and GSH in the serum were determined using commercial assay kits obtained from Nanjing Jiancheng Bioengineering Institute (Nanjing, China) according to the manufacturer's instructions.

The activity of SOD was assayed using the xanthine oxidase method based on the generation of superoxide ions, which react with water-soluble tetrazolium to form a red formazan dye. Following reaction for $20 \mathrm{~min}$ at $37^{\circ} \mathrm{C}$, the absorbance at $450 \mathrm{~nm}$ was detected using a plate reader, and the values were calculated and expressed as $\mathrm{U} / \mathrm{ml}$.

The activity of GSH-Px was determined based on its catalyzation on the conversion of GSH to oxidized glutathione (GSSG) (24). The change in absorbance at $412 \mathrm{~nm}$ during the conversion of GSH to GSSG was measured using a spectrophotometer (UV752; Yoke Instrument Co., Ltd., Shanghai, China). A single unit of GSH-Px activity was defined as the quantity of enzyme to oxidize $1 \mu \mathrm{mol} / \mathrm{l} \mathrm{GSH}$ in a reaction system at $37^{\circ} \mathrm{C}$ for $5 \mathrm{~min}$ in $0.1 \mathrm{ml}$ serum following deduction of the role of non-enzymatic reactions, and expressed in $\mathrm{U} / \mathrm{ml}$.

The activity of GSH was determined based on the principle that GSH can react with 5,5'-dithiobis (2-nitrobenzoic acid) to generate a yellow substance. The absorbance at $405 \mathrm{~nm}$ was detected using a plate reader, and the levels of GSH were calculated and expressed in $\mathrm{nmol} / \mathrm{ml}$.

Extraction of total proteins, cytoplasmic proteins and nuclear proteins. For the extraction of total proteins, the synovial tissues were sectioned and lysed in RIPA buffer (Beyotime Institute of Biotechnology, Jiangsu, China) supplemented with PMSF on ice for $5 \mathrm{~min}$. Following centrifugation of the lysates at $12,000 \mathrm{~g}$ for $10 \mathrm{~min}$ at $4^{\circ} \mathrm{C}$, the supernatant was collected as the total lysate protein and stored at $-70^{\circ} \mathrm{C}$.

The cytoplasmic and nuclear proteins were extracted from the synovial tissues using a nuclear and cytoplasmic protein extraction kit (Beyotime Institute of Biotechnology), according to the manufacturer's protocols. Briefly, the synovial tissues were cut into small sections and homogenized with cytoplasmic protein extraction agents. Following incubation on ice for $15 \mathrm{~min}$ and centrifugation at $1,500 \mathrm{~g}$ for $5 \mathrm{~min}$ at $4^{\circ} \mathrm{C}$, the supernatant was collected as partial cytoplasmic proteins and the pellets were re-suspended in cytoplasmic extraction buffer. Following incubation on ice for $15 \mathrm{~min}$ and centrifugation at $12,000 \mathrm{~g}$ for $5 \mathrm{~min}$ at $4^{\circ} \mathrm{C}$, the supernatant was combined with the above cytoplasmic protein and stored at $-70^{\circ} \mathrm{C}$. Nuclear pellets were then re-suspended in nuclear extraction buffer and vigorously shaken for $30 \mathrm{~min}$ at $4^{\circ} \mathrm{C}$. Following centrifugation at $12,000 \mathrm{~g}$ for $10 \mathrm{~min}$ at $4^{\circ} \mathrm{C}$, the nuclear proteins were obtained and stored at $-70^{\circ} \mathrm{C}$ until use. The concentrations of total proteins, cytoplasmic extracts and nuclear extracts were quantified using a BCA Protein Assay kit (Beyotime Institute of Biotechnology), according to the manufacturer's protocol.

Western blot analysis. The total, cytoplasmic and nuclear protein extracts $(40 \mu \mathrm{g})$ were separated on $8-10 \%$ SDS-PAGE 
gels. Following electrophoresis, the separated proteins were transferred onto polyvinylidene difluoride membranes (EMD Millipore, Billerica, MA, USA), and blocked with 5\% nonfat milk in Tris-buffered saline with Tween 20 for $1 \mathrm{~h}$ at room temperature. The membranes were then incubated overnight at $4^{\circ} \mathrm{C}$ with the appropriate primary antibodies, as follows: Rabbit polyclonal NALP1 (1:200; cat. no. sc-66993), goat polyclonal NALP6 (1:200; cat. no. sc-50634) from Santa Cruz Biotechnology, Inc. (Dallas, TX, USA), inhibitor of

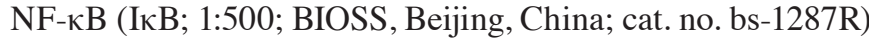
and NF-кB p65 (1:400; Boster, Inc., Wuhan, China; cat. no. BA0610). Following washing, the blots were incubated with secondary horseradish peroxidase-conjugated goat anti-rabbit (1:5,000; cat. no. A0208) or donkey anti-goat IgG (1:5,000; Beyotime Institute of Biotechnology; cat. no. A0181) for $45 \mathrm{~min}$ at $37^{\circ} \mathrm{C}$. Subsequently, the blots of interest were visualized using enhanced chemiluminescence (ECL; 7Sea Biotech, Shanghai, China) and the band densities were quantified using a Gel-Pro-Analyzer system (Liuyi Instrument Factory, Beijing, China). The expression of each protein was standardized by stripping the blots and re-probing with mouse monoclonal $\beta$-actin antibody (1:1,000; Santa Cruz Biotechnology, Inc.; sc-47778) or rabbit polyclonal histone H3 (1:200; BIOSS; cat. no. bs-17422R).

Statistical analysis. All data are presented as the mean \pm standard deviation and all statistical analyses were performed using GraphPad Prism software (version 5.0; GraphPad Software Inc., La Jolla, CA). One-way analysis of variance was used to analyze significant differences between the groups, followed by Newman-Keul's test. $\mathrm{P}<0.05$ was considered to indicate a statistically significant difference.

\section{Results}

Effects of ZSD on MSU crystal-induced paw edema in rats. As shown in Fig. 1A and B, MSU crystals led to a significant increase in the ankle diameter of the rats, compared with the normal control rats. Of note, treatment with ZSD (20 and $40 \mathrm{mg} / \mathrm{kg}$ ) significantly suppressed the MSU crystal-induced ankle swelling, and this effect was comparable with that of the positive control drug, colchicine.

ZSD improves histopathological damage in rats with gouty arthritis. To observe the effects of ZSD on the histopathology of the synovium, rat knee joint capsules were removed and analyzed using H\&E staining. As shown in Fig. 2, compared with the control rats, the sections from the MSU model rats showed marked infiltration of inflammatory cells and a markedly thickened synovium, whereas this inflammatory response and synovial hyperplasia was attenuated by treatment with ZSD, which occurred in a dose-dependent manner. Of note, the high dose ZSD (40 mg/kg) exerted a similar effect as the positive control drug, colchicine.

ZSD decreases the levels of $I L-1 \beta$ and $T N F-\alpha$ in $M S U$ crystal-induced rats. It is well accepted that pro-inflammatory cytokines, including IL- $1 \beta$ and TNF- $\alpha$ are central to the initiation and propagation of MSU crystal-induced gouty arthritis $(25,26)$. Consistent with a previous report $(27)$, the
A

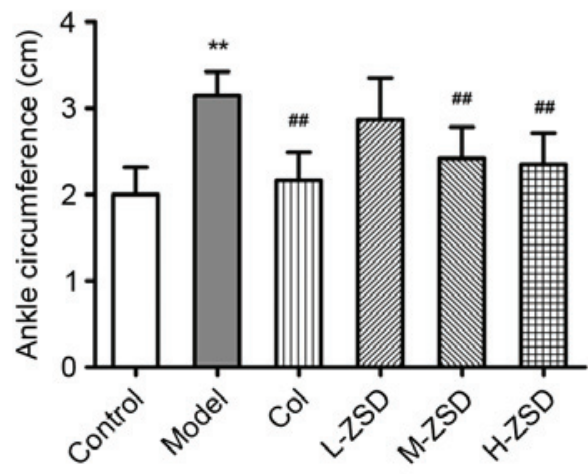

B

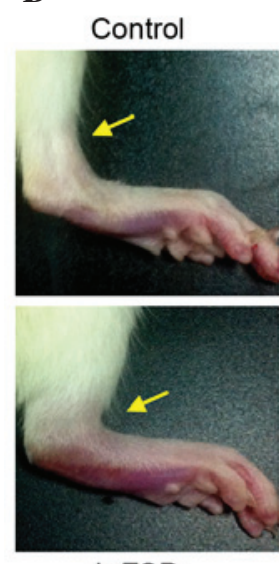

L-ZSD

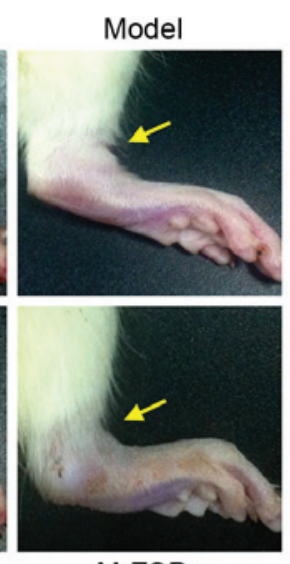

M-ZSD

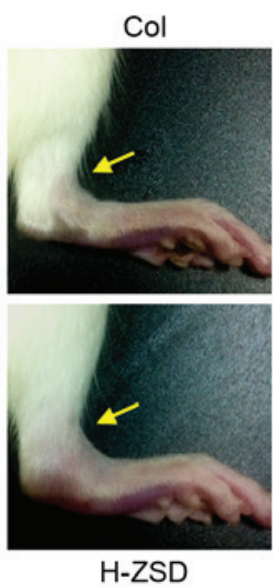

Figure 1. Effect of ZSD on ankle oedema in rats with MSU crystal-induced gouty arthritis. The treatment groups were as follows: Control=normal control rats; Model=MSU crystal-induced rats; $\mathrm{Col}=\mathrm{MSU}$ crystal-induced rats pretreated with colchicine $(0.28 \mathrm{mg} / \mathrm{kg}) ; \mathrm{L}-\mathrm{ZSD}=\mathrm{MSU}$ crystal-induced rats pretreated with ZSD $(10 \mathrm{mg} / \mathrm{kg})$; M-ZSD=MSU crystal-induced rats pretreated with ZSD $(20 \mathrm{mg} / \mathrm{kg}) ; \mathrm{H}-\mathrm{ZSD}=\mathrm{MSU}$ crystal-induced rats pretreated with ZSD (40 mg/kg). (A) Measurement of the left ankle circumference of each rat was recorded following MSU injection for 24 h. (B) Representative images of the left leg from each group are shown. Arrows indicate the position of the measurement. Data are presented as the mean \pm standard deviation $(\mathrm{n}=6) .{ }^{* *} \mathrm{P}<0.01$, vs. control group; ${ }^{\# \#} \mathrm{P}<0.01$, vs. model group. ZSD, Zisheng Shenqi decoction; MSU, monosodium urate; Col, colchicine.

MSU crystals caused a marked elevation in the levels of IL-1 $\beta$ and TNF- $\alpha$ in the serum of the rats (Fig. 3). By contrast, these increased levels of IL-1 $\beta$ and TNF- $\alpha$ were found to be significantly inhibited by ZSD (20 and $40 \mathrm{mg} / \mathrm{kg}$ ) administration and colchicine treatment.

ZSD suppresses the activation of $N F-\kappa B$ in the synovium of rats with gouty arthritis. The transcription factor, $\mathrm{NF}-\kappa \mathrm{B}$, is essential during inflammatory responses, and the activation of $\mathrm{NF}-\kappa \mathrm{B}$ has been identified as a key step in the pathogenesis of gouty arthritis (28). Therefore, the present study also investigated the effect of ZSD on the expression levels of NF- $\kappa \mathrm{B}$ p65 and its cytoplasmic inhibitory protein, $I_{\kappa} B$, in the synovium of MSU crystal-induced rats using western blot analysis. As shown in Fig. 4, the expression levels of nuclear NF- $\kappa \mathrm{B}$ p65 in the MSU crystal model rats were significantly higher, compared with those in the control group, whereas the protein levels of $\mathrm{I} \kappa \mathrm{B}$ in the cytoplasm were significantly reduced by MSU crystal injection, suggesting that MSU crystals caused 


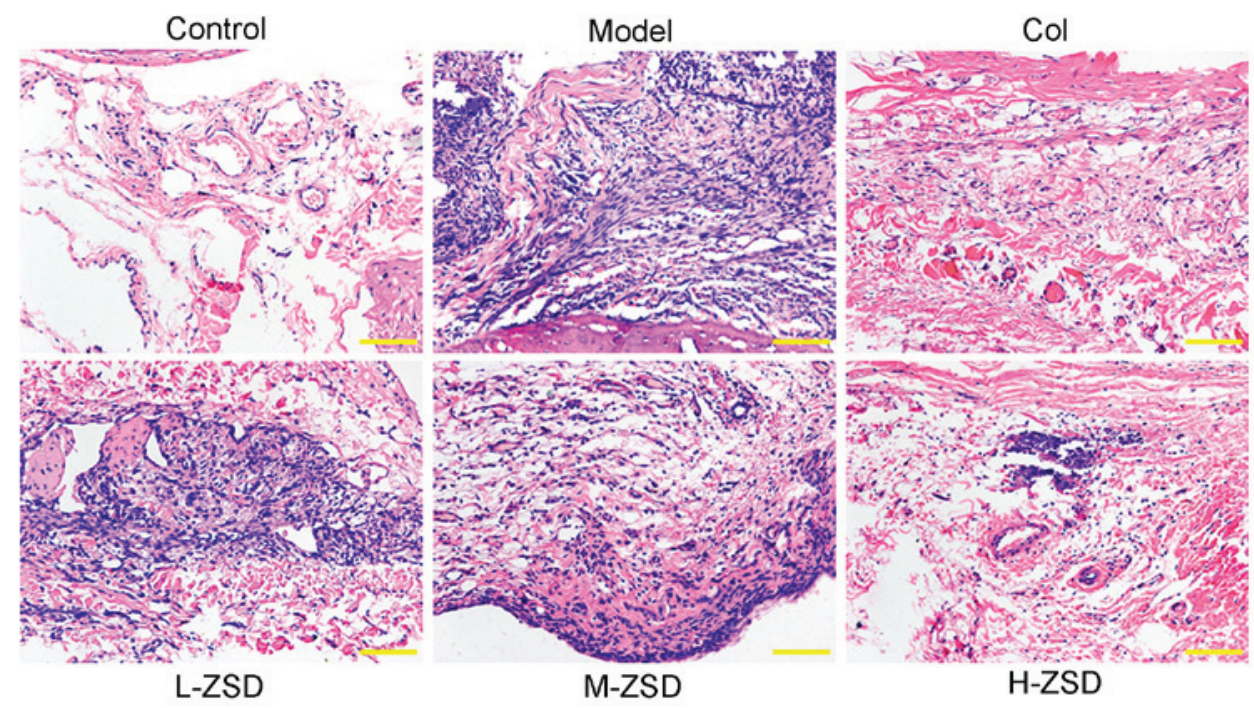

Figure 2. Effect of ZSD on MSU crystal-induced synovial damage and inflammatory cell infiltration in rats with gouty arthritis rats. Representative images of sections of the synovium from control rats, model rats, Col-treated rats and ZSD (10,20 and $40 \mathrm{mg} / \mathrm{kg}$, respectively)-treated rats stained with hematoxylin and eosin for histological assessment. Original magnification. x200. ZSD, Zisheng Shenqi decoction; MSU, monosodium urate; Col, colchicine.
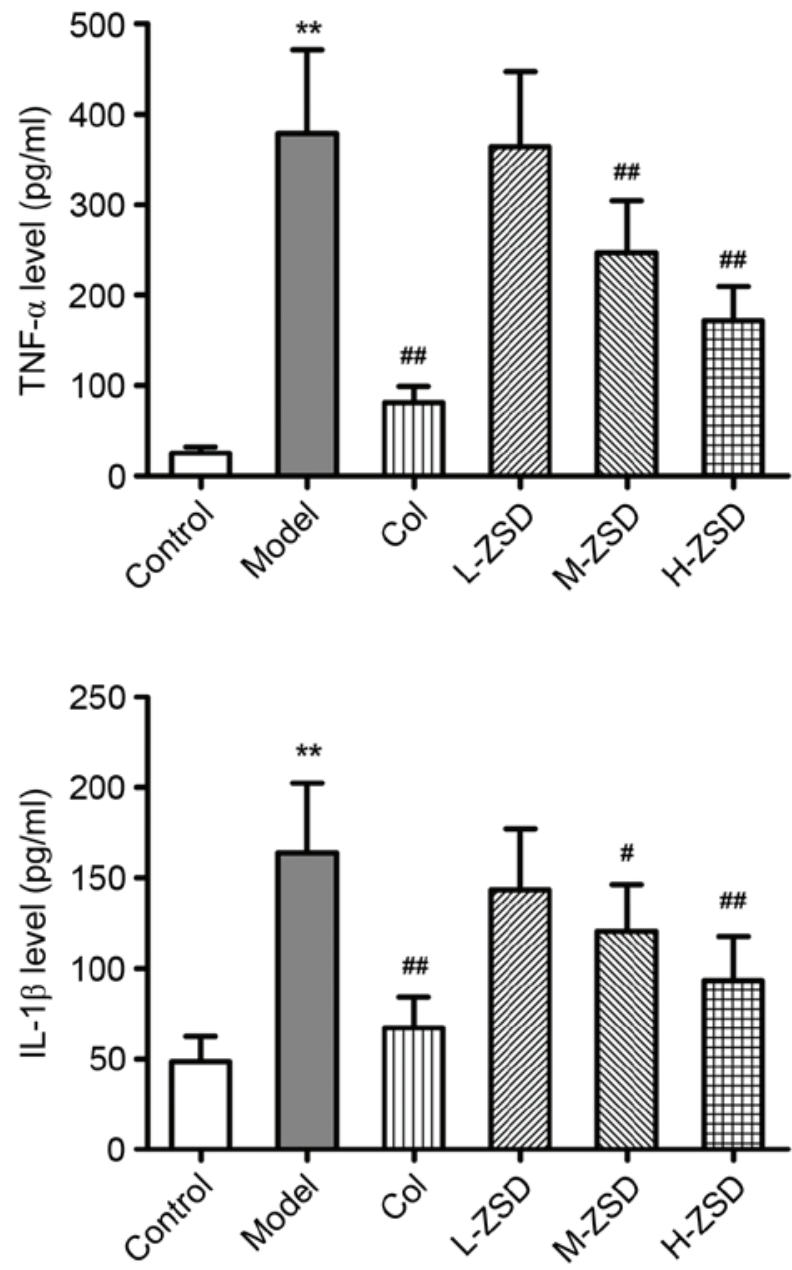

Figure 3. Effect of ZSD on serum levels of TNF- $\alpha$ and IL-1 $\beta$ in rats with MSU crystal-induced gouty arthritis. The serum levels of TNF- $\alpha$ and IL-1 $\beta$ in each rat from control group, model group, $\mathrm{Col}$ treatment group and ZSD treatment groups were determined using enzyme-linked immunosorbent assay. Data are presented as the mean \pm standard deviation $(\mathrm{n}=6) .{ }^{* *} \mathrm{P}<0.01$. vs. control group; ${ }^{\#} \mathrm{P}<0.05$ and ${ }^{\# \#} \mathrm{P}<0.01$, vs. model group. ZSD, Zisheng Shenqi decoction; MSU, monosodium urate; TNF- $\alpha$, tumor necrosis factor- $\alpha$; IL-1 $\beta$, interleukin-1 $\beta$; Col, colchicine.
$\mathrm{NF}-\kappa \mathrm{B}$ activation in the synovium. The oral administration of ZSD (40 mg/kg) inhibited the upregulation of nuclear p65 protein and downregulation of cytoplasmic I $\mathrm{B}$ protein. These results indicated that ZSD suppressed the nuclear translocation and activation of NF- $\kappa \mathrm{B}$ p65 induced by MSU crystals.

Detection of the protein expression of NALP1 and NALP6 in the synovium of rats with gouty arthritis rats and the effects of ZSD. NALP1 and NALP6, two important members of the NALP family, have been shown to positively regulate the production of IL- $1 \beta$ and promote the activation of $N F-\kappa B$ in several inflammatory disorders $(29,30)$. However, whether these two inflammasomes are also involved in MSU crystal-induced gouty arthritis remains to be elucidated. The results of the present study demonstrated that the injection of MSU crystals significantly upregulated the protein expression levels of NALP1 and NALP6, compared with the control group (Fig. 5). Of note, treatment with ZSD at 20 and $40 \mathrm{mg} / \mathrm{kg}$ inhibited the upregulation of NALP1 and NALP6 induced by the MSU crystals. Colchicine also exerted a significant inhibitory effect on the expression of these two inflammasomes. These findings suggested that NALP1 and NALP6 inflammasomes were activated in the synovium of rats with gouty arthritis, and their activation was inhibited by treatment with ZSD (40 mg/kg) or colchicine.

Effects of ZSD on the anti-oxidant status of the serum of $M S U$ crystal-induced rats. There is substantial evidence to suggest that ROS overproduction and oxidative stress are also critical for the pathogenesis of gouty arthritis $(8,31)$. In order to evaluate whether ZSD also had an anti-oxidative effect in the experimental model used in the present study, the activities of SOD and GSH-Px, and the levels of GSH were determined. As shown in Fig. 6, MSU injection led to significant decreases in the activities of SOD and GSH-Px, and in the levels of GSH, compared with the control group. However, treatment with ZSD (40 mg/kg) markedly enhanced the anti-oxidant status in 

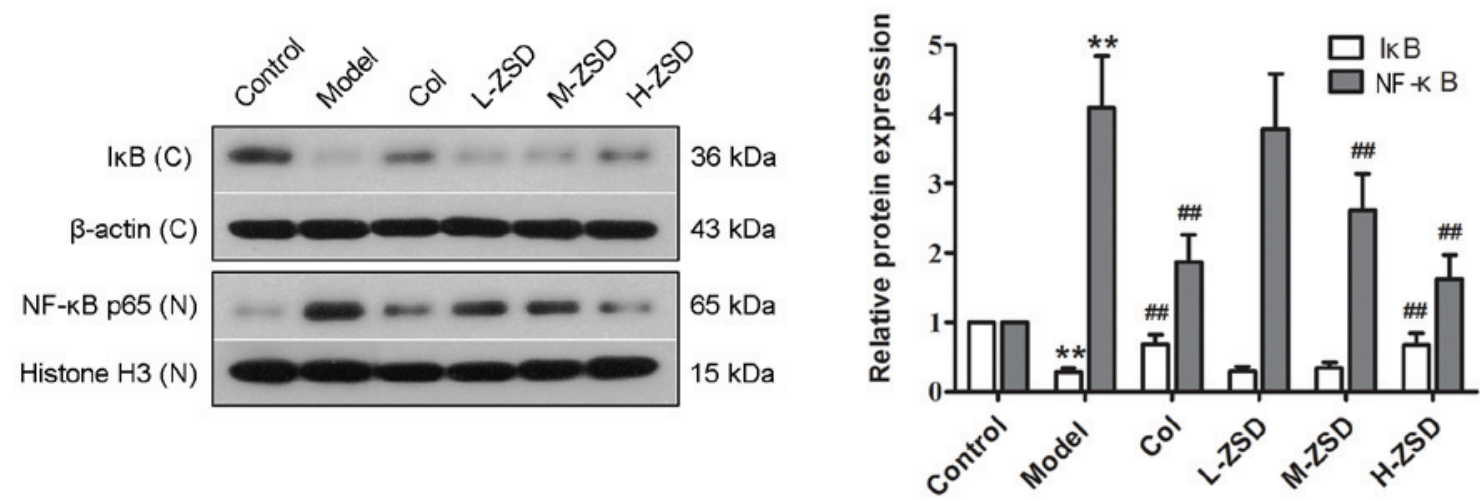

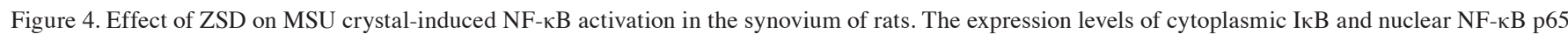
in synovial tissues from each treatment group were measured using western blot analysis. Representative bands are shown (left), and the relative band intensity ratio was analyzed (right). Data are presented as the mean \pm standard deviation $(\mathrm{n}=6) .{ }^{* *} \mathrm{P}<0.01$, vs. control group; ${ }^{* \#} \mathrm{P}<0.01$, vs. model group. $\mathrm{C}$, cytoplasm; $\mathrm{N}$,

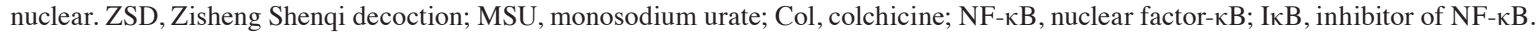
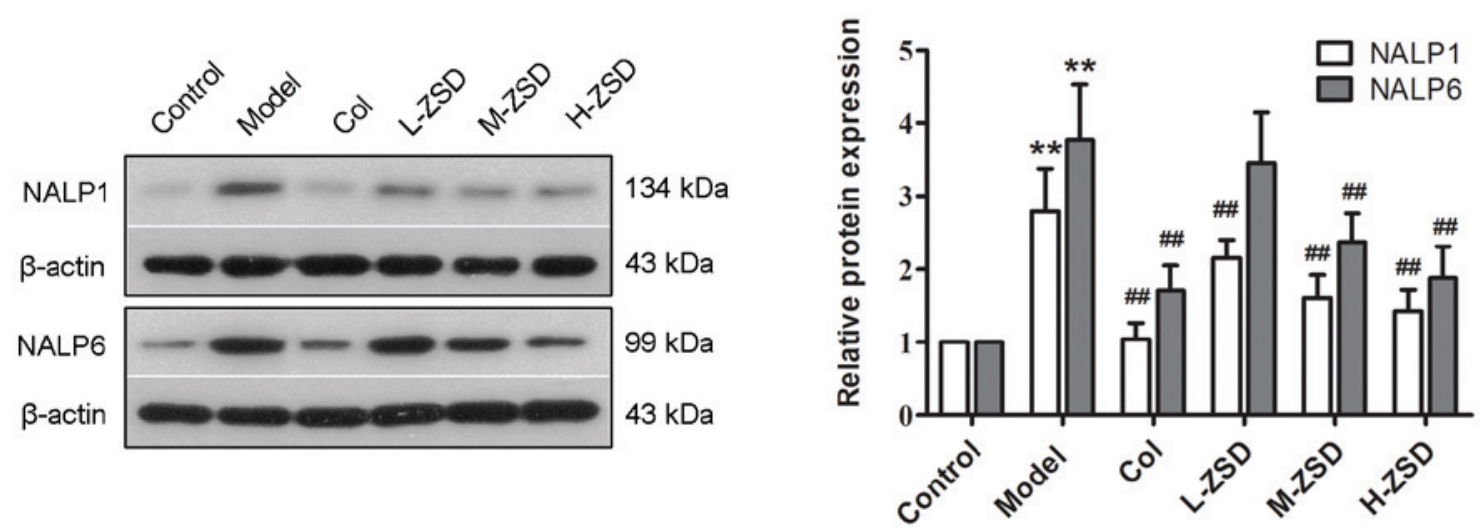

Figure 5. Effect of ZSD on the protein expression levels of NALP1 and NALP6 in the synovium of rats with MSU crystal-induced gouty arthritis. The expression levels of NALP1 and NALP6 in synovial tissues from each group were determined using western blot analysis. Representative bands are shown (left), and the relative band intensity ratio was analyzed (right). Data are presented as the mean \pm standard deviation $(\mathrm{n}=6) .{ }^{* *} \mathrm{P}<0.01$, vs. control group; ${ }^{\# \#} \mathrm{P}<0.01$, vs. model group. ZSD, Zisheng Shenqi decoction; MSU, monosodium urate; Col, colchicine; NALP, NACHT domain, leucine-rich repeat and pyrin domain containing protein.

the MSU crystal-induced rats, indicating its anti-peroxidative effect.

\section{Discussion}

With continuous improvements in living standards, the incidence of gouty arthritis has risen. Increasing interest in treating gouty arthritis has led to a focus on complementary and alternative medicine (CAM) (32). Existing evidence suggests that traditional Chinese medicines, as one of the most popular CAM products, have beneficial efficacy in the prevention and treatment of gouty arthritis $(8,27)$. In the present study, the effect of a novel herbal formula, ZSD, against gouty arthritis were examined in rats with MSU crystal-induced gouty arthritis rats, and its potential mechanism was investigated.

A clinically definitive diagnosis of gouty arthritis is based on the identification of MSU crystals in the synovium or joint fluid (33). The experimental model used in the present study represents a well-established model of gouty arthritis, induced by the injection of MSU crystals in rats. The most significant symptom of gouty arthritis is ankle swelling, which was observed in the model group following $24 \mathrm{~h}$ of MSU injection. Of note, ZSD pretreatment (40 mg/kg) markedly prevented the MSU crystal-induced elevation in ankle swelling. It is well established that the recruitment and infiltration of neutrophils into the joint fluid and synovium is a primary hallmark of gouty arthritis (34). Following the activation of monocytes and neutrophils, these cells actively phagocytoze MSU crystals, which subsequently trigger the inflammatory caspase responses. Therefore, inhibiting inflammatory cell infiltration may be an effective therapeutic strategy against gouty arthritis. The histopathology results in the present study demonstrated that a ZSD dose of $40 \mathrm{mg} / \mathrm{kg}$ significantly attenuated the inflammatory cell infiltration into the synovium induced by MSU crystals and improved synovial hyperplasia. These findings suggested that ZSD protected the rats from MSU crystal-induced gouty arthritis and synovial damage.

It is also well known that gouty arthritis is an inflammatory disease resulting from the deposition of MSU crystals in the joints. The mechanisms underlying this inflammatory cascade caused by MSU crystals have been under investigation for several years, and a number of studies have demonstrated that pro-inflammatory cytokines, including IL-1 $\beta$ and TNF- $\alpha$, and the transcription factor, $\mathrm{NF}-\kappa \mathrm{B}$, are important in response to the injection of MSU crystals into the joint cavity $(35,36)$. In the pathogenesis of gouty arthritis, $N F-\kappa B$ signals can 

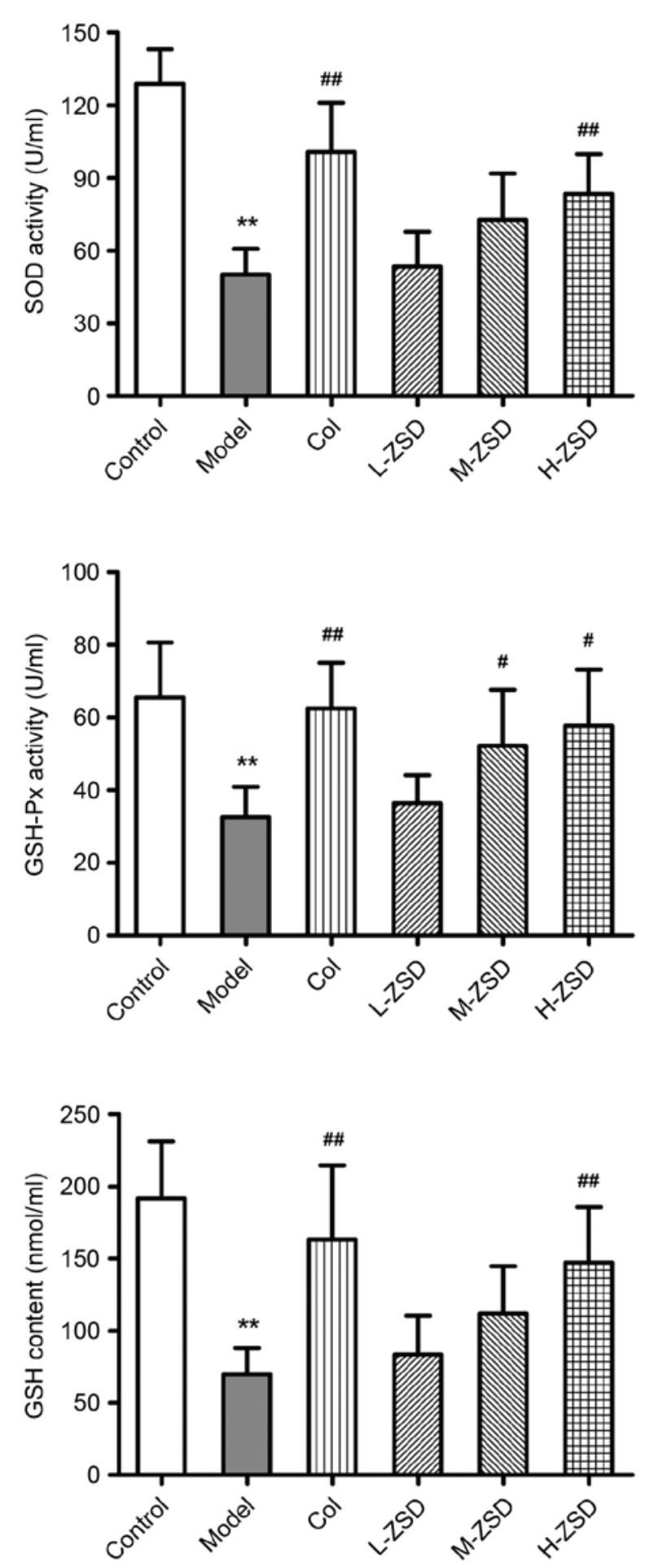

Figure 6. Effect of ZSD on the anti-oxidant status of serum obtained from rats with MSU crystal-induced gouty arthritis. The activities of SOD and GSH-Px, and the levels of GSH in the serum of each rat from the control group, model group, $\mathrm{Col}$ treatment group and ZSD treatment groups were measured. The data are presented as the mean \pm standard deviation $(n=6)$. ${ }^{* *} \mathrm{P}<0.01$, vs. control group; ${ }^{\#} \mathrm{P}<0.05$ and ${ }^{\# \#} \mathrm{P}<0.01$; vs. model group. ZSD, Zisheng Shenqi decoction; MSU, monosodium urate; Col, colchicine; SOD; superoxide dismutase; GSH-Px, glutathione peroxidase; GSH, reduced glutathione.

stimulate the production of genes encoding pro-inflammatory cytokines. Conversely, the overexpression of TNF- $\alpha$ and IL-1 $\beta$ can directly activate the $\mathrm{NF}-\kappa \mathrm{B}$ pathway, leading to a positive feedback loop, which further amplifies inflammatory responses and causes joint injury (35). Normally, NF- $\kappa \mathrm{B}$ binds to an inhibitory protein, $\mathrm{I} \kappa \mathrm{B}$, and localizes in the cytoplasm. Certain stimuli, including MSU crystals, can lead to the degradation of $\mathrm{I} \kappa \mathrm{B}$ and translocation of $\mathrm{NF}-\kappa \mathrm{B}$ into the nucleus where it regulates the transcription of various target genes (37). In the present study, the levels of IL- $1 \beta$ and TNF- $\alpha$ in the serum were significantly increased in response to MSU crystals, whereas this overproduction was markedly decreased by ZSD treatment, similar to the positive drug, colchicine. Additionally, the findings in the present study demonstrated that ZSD prevented the MSU crystal-induced nuclear translocation and activation of NF- $\kappa \mathrm{B}$ p65 in the synovium. Taken together, these results suggested that ZSD attenuated MSU crystal-induced gouty arthritis via anti-inflammatory action, which may be associated with interrupting the positive feedback loop between the NF- $\kappa \mathrm{B}$ pathway and cytokines, including IL-1 $\beta$ and TNF- $\alpha$.

The process involved in the production of IL-1 $\beta$ in response to MSU crystals is complex, and increasing evidence has shown that NALP inflammasomes are pivotal in this process. There is evidence that MSU stimulation triggers the activation of the NALP3 inflammasome, one of the most well-characterized NALP family members in leukocytes, which in turn converts procaspase- 1 to active caspase- 1 , which cleaves pro-IL-1 $\beta$ to the active secreted form, IL-1 $\beta$ (38). In addition, previous studies have demonstrated that colchicine can reduce NALP3 inflammasome-driven caspase-1 activation by preventing microtubule assembly and MSU delivery (39). Similar to NALP3, other members of the NALP family, including NALP1 and NALP6, have also been identified as triggers of caspase-1 activation in the pathogenesis of several inflammatory diseases $(29,30,40)$. The studies by Zhu et al (29) and Wang et al (41) demonstrated that the NALP1 inflammasome is involved in the inflammatory reaction process of rheumatoid arthritis by activating caspase-1. Additionally, NALP6 possesses structural motifs similar to those of molecular sensors, leading to pro-caspase-1 activation with NALP3 (42). However, whether NALP1 and NALP6 inflammasomes are involved in the pathogenesis of gouty arthritis has not been reported to date, to the best of our knowledge. In the present study, it was demonstrated that the injection of MSU crystals into the joint cavity caused marked activation of the NALP1 and NALP6 inflammasomes in the synovium. Of note, their activation by MSU crystals was suppressed by treatment with ZSD (20 and $40 \mathrm{mg} / \mathrm{kg}$ ) or colchicine. These findings revealed that the NALP1 and NALP6 inflammasomes may be relevant therapeutic targets in the treatment of gouty arthritis. However, further investigation of the mechanisms and the detailed regulatory effect of ZSD on these mechanisms are required.

A previous report revealed that oxidative stress and ROS are important in the activation of NALP inflammasomes induced by MSU crystals (36). In phagocytic cells, the free radicals and ROS can cause damage to the cells if maintained at higher levels than normal. In order to circumvent this damage, several defense mechanisms, including SOD, GSH-Px and catalase, are initiated (31). In agreement with previous reports $(25,31)$, the results of the present study showed that the activities of SOD and GSH-Px, and the levels of GSH were significantly decreased in the rats with MSU crystal-induced gouty arthritis, compared with the rats in the control group, which may be due to their increased consump- 
tion in response to oxidative stress. Of note, ZSD treatment $(40 \mathrm{mg} / \mathrm{kg})$ markedly enhanced the anti-oxidant status in the MSU crystal-induced rats. These findings suggested that ZSD can also prevent MSU crystal-induced gouty arthritis via anti-oxidative effects.

In conclusion, the present study demonstrated for the first time, to the best of our knowledge, that ZSD, a novel Chinese herbal formula with multiple functions in nourishing kidney and removing dampness, effectively prevented gouty arthritis in the MSU crystal-induced rat model. The mechanisms involved in this effect of ZSD on gouty arthritis were associated with the modulation of multiple anti-oxidant and anti-inflammatory pathways, as evidenced by increasing activities of SOD and GSH-Px, increased levels of GSH, downregulation of NALP1 and NALP6 inflammasomes, reductions in the IL-1 $\beta$ and TNF- $\alpha$ pro-inflammatory cytokines, and inhibition of the activation of NF- $\mathrm{KB}$. These results suggested that ZSD may be a promising therapeutic formula for the prevention and treatment of gouty arthritis in a clinical setting.

\section{Acknowledgements}

This study was supported by a grant from the National Natural Science Foundation of China (grant no. 81173170).

\section{References}

1. Agudelo CA and Wise CM: Gout: Diagnosis, pathogenesis, and clinical manifestations. Curr Opin Rheumatol 13: 234-239, 2001.

2. Woolf AD and Dieppe PA: Mediators of crystal-induced inflammation in the joint. Br Med Bull 43: 429-444, 1987.

3. Margalit A, Duffin KL, Shaffer AF, Gregory SA and Isakson PC: Altered arachidonic acid metabolism in urate crystal induced inflammation. Inflammation 21: 205-222, 1997.

4. Martinon F, Pétrilli V, Mayor A, Tardivel A and Tschopp J: Gout-associated uric acid crystals activate the NALP3 inflammasome. Nature 440: 237-241, 2006.

5. Ogura Y, Sutterwala FS and Flavell RA: The inflammasome: First line of the immune response to cell stress. Cell 126 659-662, 2006.

6. Giamarellos-Bourboulis EJ, Mouktaroudi M, Bodar E, van der Ven J, Kullberg BJ, Netea MG and van der Meer JW: Crystals of monosodium urate monohydrate enhance lipopolysaccharide-induced release of interleukin 1 beta by mononuclear cells through a caspase 1-mediated process. Ann Rheum Dis 68 : 273-278, 2009.

7. Schlesinger $N$ and Thiele RG: The pathogenesis of bone erosions in gouty arthritis. Ann Rheum Dis 69: 1907-1912, 2010.

8. Murunikkara V and Rasool M: Trikatu, a herbal compound that suppresses monosodium urate crystal-induced inflammation in rats, an experimental model for acute gouty arthritis. Cell Biochem Funct 32: 106-114, 2014.

9. Fang $\mathrm{ZH}$ and Waizy H: Current concepts in the treatment of gouty arthritis. Orthop Surg 5: 6-12, 2013.

10. Fam AG: Treating acute gouty arthritis with selective COX 2 inhibitors. BMJ 325: 980-981, 2002.

11. Li XX, Han M, Wang YY and Liu JP: Chinese herbal medicine for gout: A systematic review of randomized clinical trials. Clin Rheumatol 32: 943-959, 2013.

12. Wilson L and Saseen JJ: Gouty arthritis: A review of acute management and prevention. Pharmacotherapy: Jun 18, 2016 (Epub ahead of print)

13. Wang W, Bhole VM and Krishnan E: Chronic kidney disease as a risk factor for incident gout among men and women: retrospective cohort study using data from the Framingham Heart Study. BMJ Open 5: e006843, 2015.

14. Sellin L, Kielstain JT and de Groot K: Hyperuricemia- more than gout: Impact on cardiovascular risk and renal insufficiency. Z Rheumatol 74: 322-328, 2015.
15. Wang Y, Wang L, Li E, Li Y, Wang Z, Sun X, Yu X, Ma L, Wang Y and Wang Y: Chuanhu anti-gout mixture versus colchicine for acute gouty arthritis: A randomized, double-blind, double-dummy, non-inferiority trial. Int J Med Sci 11: 880-885, 2014.

16. Wu CR, Lin LW, Wang WH and Hsieh MT: The ameliorating effects of LiuWei Dihuang Wang on cycloheximide-induced impairment of passive avoidance performance in rats. J Ethnopharmacol 113: 79-84, 2007.

17. Lian F, Wu HC, Sun ZG, Guo Y, Shi L and Xue MY: Effects of Liuwei Dihuang Granule ([symbols; see text]) on the outcomes of in vitro fertilization pre-embryo transfer in infertility women with Kidney-yin deficiency syndrome and the proteome expressions in the follicular fluid. Chin J Integr Med 20: 503-509, 2014.

18. Qiu R, Shen R, Lin D, Chen Y and Ye H: Treatment of 60 cases of gouty arthritis with modified Simiao Tang. J Tradit Chin Med 28: 94-97, 2008.

19. Jiang J, Wu F, Lu J, Lu Z and Xu Q: Anti-inflammatory activity of the aqueous extract from Rhizoma smilacis glabrae. Pharmacol Res 36: 309-314, 1997

20. Jiang Y, Zhang Y, Chen W, Liu C, Li X, Sun D, Liu Z, Xu Y, Mao X, Guo Q and Lin N: Achyranthes bidentata extract exerts osteoprotective effects on steroid-induced osteonecrosis of the femoral head in rats by regulating RANKL/RANK/OPG signaling. J Transl Med 12: 334, 2014.

21. Chiang LC, Chiang W, Chang MY and Lin CC: In vitro cytotoxic, antiviral and immunomodulatory effects of Plantago major and Plantago asiatica. Am J Chin Med 31: 225-234, 2003.

22. Huang Q, Duan Z, Yang J, Ma X, Zhan R, Xu H and Chen W: SNP typing for germplasm identification of Amomum villosum Lour. Based on DNA barcoding markers. PloS One 9: e114940, 2014

23. Coderre TJ and Wall PD: Ankle joint urate arthritis (AJUA) in rats: An alternative animal model of arthritis to that produced by Freund's adjuvant. Pain 28: 379-393, 1987.

24. Li HX, Xiao Y, Cao LL, Yan X, Li C, Shi HY, Wang JW and Ye YH: Cerebroside $\mathrm{C}$ increases tolerance to chilling injury and alters lipid composition in wheat roots. PloS One 8: e73380, 2013.

25. Sabina EP, Nagar S and Rasool M: A role of piperine on monosodium urate crystal-induced inflammation-an experimental model of gouty arthritis. Inflammation 34: 184-192, 2011.

26. de Souza MR, de Paula CA, Pereira de Resende ML, Grabe-Guimarães A, de Souza Filho JD and Saúde-Guimãraes DA: Pharmacological basis for use of Lychnophora trichocarpha in gouty arthritis: Anti-hyperuricemic and anti-inflammatory effects of its extract, fraction and constituents. J Ethnopharmacol 142: 845-850, 2012.

27. Jiang Y, You XY, Fu KL and Yin WL: Effects of extract from Mangifera indica Leaf on monosodium urate crystal-induced gouty arthritis in rats. Evid Based Complement Alternat Med 2012: 967573, 2012.

28. Jaramillo M, Godbout M, Naccache PH and Olivier M: Signaling events involved in macrophage chemokine expression in response to monosodium urate crystals. J Biol Chem 279: 52797-52805, 2004.

29. Zhu L, Li J, Guo L, Yu X, Wu D, Luo L, Zhu L, Chen W, Chen C, Ye C and Zhang D: Activation of NALP1 inflammasomes in rats with adjuvant arthritis; a novel therapeutic target of carboxyamidotriazole in a model of rheumatoid arthritis. Br J Pharmacol 172: 3446-3459, 2015.

30. Grenier JM, Wang L, Manji GA, Huang WJ, Al-Garawi A, Kelly R, Carlson A, Merriam S, Lora JM, Briskin M, et al: Functional screening of five PYPAF family members identifies PYPAF5 as a novel regulator of NF-kappaB and caspase-1. FEBS Lett 530: 73-78, 2002.

31. Sabina EP, Rasool M, Mathew L, Ezilrani P and Indu H: 6-Shogaol inhibits monosodium urate crystal-induced inflammation-an in vivo and in vitro study. Food Chem Toxicol 48: 229-235, 2010.

32. Venkatesha SH, Rajaiah R, Berman BM and Moudgil KD: Immunomodulation of autoimmune arthritis by herbal CAM. Evid Based Complement Alternat Med 2011: 986797, 2011.

33. Schlesinger N: Diagnosing and treating gout: A review to aid primary care physicians. Postgrad Med 122: 157-161, 2010.

34. Torres R, Macdonald L, Croll SD, Reinhardt J, Dore A, Stevens S, Hylton DM, Rudge JS, Liu-Bryan R, Terkeltaub RA, et al: Hyperalgesia, synovitis and multiple biomarkers of inflammation are suppressed by interleukin 1 inhibition in a novel animal model of gouty arthritis. Ann Rheum Dis 68: 1602-1608, 2009.

35. Ma Y, Zhou LL, Yan HY and Liu M: Effects of extracts from Paederia scandens (LOUR.) MERRILL (Rubiaceae) on MSU crystal-induced rats gouty arthritis. Am J Chin Med 37: 669-683, 2009. 
36. Punzi L, Scanu A, Ramonda R and Oliviero F: Gout as autoinflammatory disease: New mechanisms for more appropriated treatment targets. Autoimmun Rev 12: 66-71, 2012.

37. Grall F, Gu X, Tan L, Cho JY, Inan MS, Pettit AR, Thamrongsak U, Choy BK, Manning C, Akbarali Y, et al: Responses to the proinflammatory cytokines interleukin-1 and tumor necrosis factor alpha in cells derived from rheumatoid synovium and other joint tissues involve nuclear factor kappaB-mediated induction of the Ets transcription factor ESE-1. Arthritis Rheum 48: 1249-1260, 2003.

38. Zheng SC, Zhu XX, Xue Y, Zhang LH, Zou HJ, Qiu JH and Liu Q: Role of the NLRP3 inflammasome in the transient release of IL-1 $\beta$ induced by monosodium urate crystals in human fibroblast-like synoviocytes. J Inflamm (Lond) 12: 30, 2015.
39. Dalbeth N, Lauterio TJ and Wolfe HR: Mechanism of action of colchicine in the treatment of gout. Clin Ther 36: 1465-1479, 2014

40. Bruey JM, Bruey-Sedano N, Luciano F, Zhai D, Balpai R, Xu C, Kress CL, Bailly-Maitre B, Li X, Osterman A, et al: Bcl-2 and Bcl-XL regulate proinflammatory caspase-1 activation by interaction with NALP1. Cell 129: 45-56, 2007.

41. Wang T, Zhu CL, Wang S, Mo LW, Yang GD, Hu J and Zhang F: Role of NLRP3 and NLRP1 inflammasomes signaling pathways in pathogenesis of rheumatoid arthritis. Asian Pac J Trop Med 7: 827-831, 2014.

42. Anand PK and Kanneganti TD: NLRP6 in infection and inflammation. Microbes Infect 15: 661-668, 2013. 